\title{
Group-Based Recurrent Neural Networks for POI Recommendation
}

\author{
GUOHUI LI, QI CHEN, and BOLONG ZHENG, Huazhong University of Science and Technology \\ HONGZHI YIN, University of Queensland \\ QUOC VIET HUNG NGUYEN, Griffith University \\ XIAOFANG ZHOU, University of Queensland
}

\begin{abstract}
With the development of mobile Internet, many location-based services have accumulated a large amount of data that can be used for point-of-interest (POI) recommendation. However, there are still challenges in developing an unified framework to incorporate multiple factors associated with both POIs and users due to the heterogeneity and implicity of this information. To alleviate the problem, this work proposes a novel group-based method for POI recommendation jointly considering the reviews, categories, and geographical locations, called the Group-based Temporal Sentiment-Aspect-Region Recurrent Neural Network (GTSAR-RNN). We divide the users into different groups and then train an individual RNN for each group with the goal of improving its pertinence. In GTSAR-RNN, we consider not only the effects of temporal and geographical contexts but also the users' sentimental opinions on locations. Experimental results show that GTSAR-RNN acquires significant improvements over the baseline methods on real datasets.
\end{abstract}

\section{CCS Concepts: • Information systems $\rightarrow$ Social recommendation;}

Additional Key Words and Phrases: POI recommendation, recurrent neural network, sentiment analysis

\section{ACM Reference format:}

Guohui Li, Qi Chen, Bolong Zheng, Hongzhi Yin, Quoc Viet Hung Nguyen, and Xiaofang Zhou. 2020. GroupBased Recurrent Neural Networks for POI Recommendation. ACM/IMS Trans. Data Sci. 1, 1, Article 3 (February 2020), 18 pages.

https://doi.org/10.1145/3343037

\section{INTRODUCTION}

With the rapid growth of available information on the Internet, users are getting in trouble with the problem of information overload. Recommendation systems become important for helping users to select interesting objects in many applications, such as social networks, e-commerce, online and reading. The point-of-interest (POI) recommendation, aiming to recommend top- $k$ POIs such that

\footnotetext{
This research is partially supported by NSFC (Grants No. 61902134, 61572215), and the Fundamental Research Funds for the Central Universities (HUST: Grants No. 2019kfyXJJS091, 2019kfyXKJC021).

Authors' addresses: G. Li, School of Software Engineering, Huazhong University of Science and Technology, Wuhan, China; email: guohuili@hust.edu.cn; Q. Chen and B. Zheng (corresponding author), School of Computer Science and Technology, Huazhong University of Science and Technology, Wuhan, China; emails: \{chenqijason, bolongzheng\}@hust.edu.cn; H. Yin and X. Zhou, University of Queensland, Brisbane, Australia; emails: h.yin1@uq.edu.au, zxf@itee.uq.edu.au; Q. V. H. Nguyen, Griffith University, Gold Coast, Australia; email: henry.nguyen@griffith.edu.au.

Permission to make digital or hard copies of all or part of this work for personal or classroom use is granted without fee provided that copies are not made or distributed for profit or commercial advantage and that copies bear this notice and the full citation on the first page. Copyrights for components of this work owned by others than ACM must be honored. Abstracting with credit is permitted. To copy otherwise, or republish, to post on servers or to redistribute to lists, requires prior specific permission and/or a fee. Request permissions from permissions@acm.org. (C) 2020 Association for Computing Machinery.

2577-3224/2020/02-ART3 \$15.00

https://doi.org/10.1145/3343037
} 
a target user will be most likely to visit under a specific spatial-temporal context, has been studied broadly in recent years [18]. As an important application in location-based services (LBSs), POI recommendation benefits not only the users for obtaining the best experience on unvisited POIs but also the service providers for promoting their services with users' feedback.

Recently, the flourish of LBS, such as Yelp, ${ }^{1}$ FourSquare,${ }^{2}$ and Facebook Places, ${ }^{3}$ provides free and public available channels for users to share their personal experiences and opinions on the visited POIs. Two kinds of data contain rich contextual knowledge-check-in and review datawhich paves a way to the POI recommendation.

The trajectories of users derived from check-in data provide the opportunity for discovering useful knowledge on their behaviors [43, 45]. Markov chain (MC)-based models [6,23] aim to predict the next behavior of a user based on his past behaviors. However, the review data enables us to analyze the sentiment information for POI recommendation [12, 29, 38]. Existing work [11, 44] improves traditional recommendation models via mining useful information from check-in and review data. However, there are no existing approaches that jointly integrate location category, spatial-temporal context, and review sentiment into a unified framework.

More recently, the techniques of the neural network are applied in POI recommendation, achieving satisfactory results. Liu et al. [17] use a common context transition matrix and several common time transition matrices to construct the RNN for each user. However, these common transition matrices become less effective when the uses have distinct behavior patterns. Moreover, if we train a specific neural network for each user, the model may suffer a data sparsity problem in the training phase. For instance, in the Yelp dataset, the average number of reviews per user is less than 10.

Therefore, to balance the model pertinence and the data sparsity problem, we propose the Group-based Temporal Sentiment-Aspect-Region Recurrent Neural Network (GTSAR-RNN) model. By incorporating the recurrent architecture with continuous sentiment and geographical effects, GTSAR-RNN divides users into different groups and then trains a neural network for each group. By combining the prediction results derived from different neural networks, GTSAR-RNN recommends the potential POIs to users. The main contributions of our work are summarized as follows:

- To the best of our knowledge, we are the first to address the problem of POI recommendation by integrating sentiment into RNNs as context information.

- To improve the pertinence of the model, we divide users into different groups according to their check-in data and construct a neural network model for the users in the same group, which is a flexible strategy that captures the users' group behaviors.

- Extensive experiments are conducted on three real datasets, which show that our proposed GTSAR-RNN significantly outperforms the state-of-the-art methods.

The rest of this article is organized as follows. Section 2 reviews the related work. Section 3 gives a detailed description of the framework. The experimental evaluations are presented in Section 4. Finally, Section 5 highlights the contributions and possible directions for future study.

\section{RELATED WORK}

Location recommendation is first designed for taxi drivers to pick up the passengers more efficiently by analyzing the real-life data collected from the Taxi Telematics system [13]. Early location recommendation systems were developed to provide the most popular locations according to public opinions such as in Cao et al. [4], which do not take a user's personalized information

\footnotetext{
${ }^{1}$ https://www.yelp.com/.

${ }^{2}$ https://foursquare.com/.

${ }^{3}$ https://www.facebook.com/places/.
} 
into consideration. To meet a user's personal needs, various kinds of personalized location recommendation approaches have been proposed. We will present an overview of the most related research, including two parts,: general POI recommendation and neural network-based POI recommendation.

\subsection{General Recommendation}

2.1.1 Collaborative Filtering-Based Methods. The collaborative filtering (CF) technique has been broadly studied for plentiful personalized recommendation tasks, including memory-based CF and model-based CF. Generally, there are two different types of memory-based CF approaches: user based [30,32] and item based [3]. The user-based CF model can be used in POI recommendation by calculating the the probability of visiting all pairwise $\mathrm{POIs}^{4}[40,41]$ for estimating the probability that a user checks in a new POI. Different from using user-item rating records in classical matrix factorization approaches, another model [20] is based on check-in frequency that quantifies users' preference on locations.

2.1.2 Context-Aware Methods. Cheng et al. [5] propose a multi-center Gaussian model to capture the geographical influence and combine the matrix factorization with social regularization to perform the location recommendation. Liu et al. [18] exploit geographical characteristics from a location perspective by considering the geographical neighborhoods of a location. Although these methods can acquire good performance in location recommendation, they just consider the geographical information. Yuan et al. [32] first define the time-aware POI recommendation and integrate the spatial and temporal factors into his model. Then, they propose a method called the Geographical-Temporal influences Aware Graph (GTAG) [33] to model check-in records, geographical influence, and temporal influence. Yin et al. [31] study the problem of real-time POI recommendation by jointly modeling user check-in behaviors and region indicators. Zhang and Wang [34] propose a novel model that considers the location, time, and social information simultaneously for the successive POI recommendation task and efficiently utilizes the merits of the collaborative retrieval.

To make full use of reviews published by users, Yang et al. [29] propose a sentiment-enhanced personalized location recommendation model by combining user preference extracted from checkins and text tips that are processed by sentiment analysis techniques. Zhao et al. [38] propose a hybrid model that is capable of capturing the interaction of different factors, including topical aspect, sentiment, and spatial information. And Feng et al. [10] propose a personalized ranking metric embedding method (PRME) that avoids drawbacks of the matrix factorization technique. However, these methods are unable to capture the underlying properties in users' sequential behaviors.

\subsection{Neural Network-Based Recommendation}

With the development of deep learning, neural networks have been widely used in many domains, such as disease diagnosis [42], translation [1], and recommendation [8]. Song et al. [25] propose a novel deep neural network-based architecture that models the combination of long-term static and short-term temporal user preferences to improve the recommendation performance. To cope with the sequential recommendation, RNNs have been used in Liu et al. [17] and Zhang et al. [36]. Liu et al. [17] first extend RNNs for location recommendation and propose a novel method called Spatial Temporal Recurrent Neural Networks (ST-RNNs), which model local temporal and spatial context. Yang et al. [27] develop a semi-supervised learning architecture that jointly learns the embeddings of users and POIs to predict both user preference over POIs. By combining the

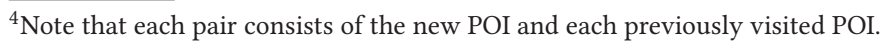


Table 1. Notations and Definitions

\begin{tabular}{c||l}
\hline Notation & \multicolumn{1}{c}{ Definition } \\
\hline \hline $\mathcal{U}, \mathcal{L}$ & User set $\mathcal{U}$ and location set $\mathcal{L}$ \\
\hline $\mathcal{V}, \mathcal{A}$ & Review set $\mathcal{V}$ and aspect set $\mathcal{A}$ \\
\hline $\mathcal{C}, \mathcal{R}$ & Category set $\mathcal{C}$ and region set $\mathcal{R}$ \\
\hline $\mathcal{T}, \mathcal{G}$ & Time bin set $\mathcal{T}$ and group set $\mathcal{G}$ \\
\hline$x_{k}^{u}$ & User $u$ 's attention on $k$-th aspect \\
\hline$y_{t_{n}, k}^{l}$ & Composite score of location $l$ on aspect $a_{k}$ \\
\hline$z_{t_{n}}^{l}$ & Users' average rating on location $l$ at $t_{n}$ \\
\hline$p_{t_{h}}^{u}$ & Representation of user $u$ at time $t_{h}$ \\
\hline$h_{t_{h}}^{u}$ & Visit embedding of user $u$ at time $t_{h}$ \\
\hline$q_{t_{h}}^{l}$ & Representation of location $l$ at time $t_{h}$ \\
\hline$W_{1}, W_{2}$ & Transition matrices for $q_{t_{p-1}}^{u}$ and $q_{t_{h}}^{u}$ \\
\hline$A, C$ & Attention and sentiment transition matrices \\
\hline$T$ & Time transition matrices \\
\hline
\end{tabular}

merits from both network embedding and sequential modeling through deep learning, Yang et al. [28] present a novel neural network model that jointly models both social networks and mobile trajectories.

Different from the work mentioned previously, we first consider sentiment, time, and geographical factors in POI recommendation at the same time. In this article, we extend the basic RNNs to learning the embeddings of different factors. The proposed GTSAR-RNN divides the users into multiple groups and train different neural networks for different groups, which increases the pertinence of the model.

\section{THE FRAMEWORK}

We first formulate the POI recommendation problem in Section 3.1 and then introduce an RNN framework, namely GTSAR-RNN, to handle the next POI recommendation task. Important notations are listed in Table 1.

\subsection{Problem Formulation}

Let $\mathcal{U}$ and $\mathcal{L}$ denote the user set and the location set, respectively, where $|\mathcal{U}|$ and $|\mathcal{L}|$ are the number of users and locations. In particular, we follow the setting in Liu et al. [17] to partition the time into discrete bins, called time bin, to study a user's visiting behavior according to a metric (e.g., day, week $)^{5}$-that is, $\mathcal{T}=\left\{\mathcal{T}_{n}\right\}_{|\mathcal{T}|}$, where $\mathcal{T}_{n}$ denotes the $n$-th time bin $\left(\right.$ i.e., $\left.\left[t_{n-1}, t_{n}\right]\right)$ and $|\mathcal{T}|$ is the number of time bins.

Given a user $u$ with a sequence of visited locations (i.e., $\mathcal{L}_{u}=\left\{l_{u, t_{1}}, l_{u, t_{2}} \ldots, l_{u, t_{h}}\right\}$ ), our task is to return a set of unvisited locations for user $u$ to explore, which can be regarded as a time sequence prediction problem.

As a state-of-the-art method, ST-RNN [17] adopts time-specific transition matrices and distancespecific transition matrices in a recurrent manner under the framework of the RNN model. NEXT [37] is another effective neural network-based model where only the latest POI visiting is taken as input. Different from these existing works that directly take only one neural network for the

\footnotetext{
${ }^{5}$ For ease of presentation, in this article, time and time bin are used interchangeably unless noted otherwise.
} 


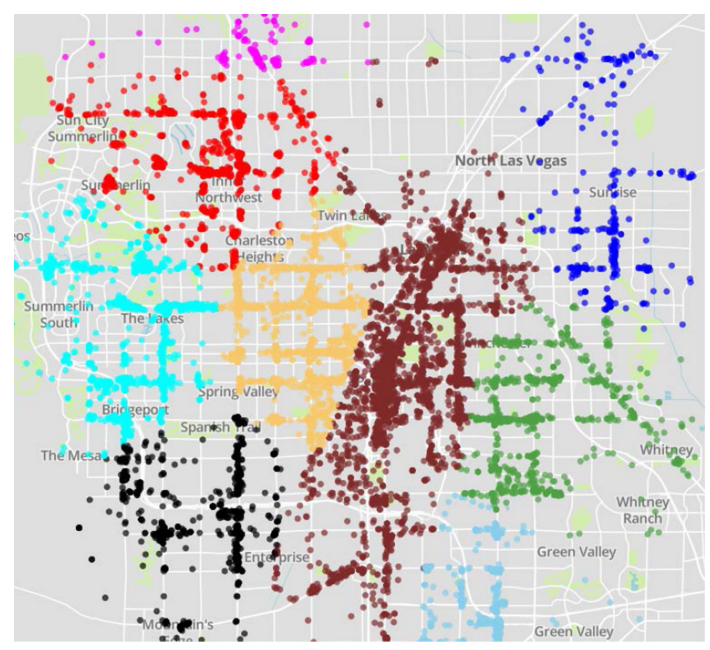

Fig. 1. Locations in Las Vegas clustered into groups.

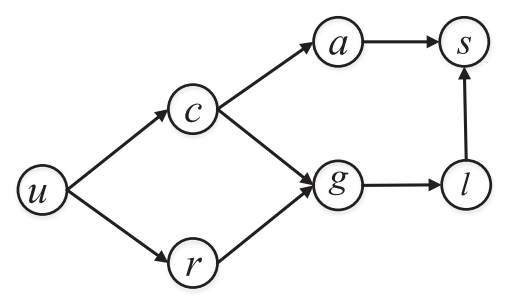

Fig. 2. Relationships in our model.

embedding calculation of users and POIs, our proposed GTSAR-RNN introduces an integration of multiple neural networks to model a user's intention. In addition, the users and POIs are always associated with auxiliary information. Therefore, in this article, we integrate user grouping, location category, spatial-temporal context, and review sentiment into a unified framework for POI recommendation.

We keep the main category tags for each location ${ }^{6}$ and use $C=\left\{c_{i}\right\}_{|C|}(|C|$ is the number of categories) to denote the category set, where $c_{i}$ represents a category, such as a bar or restaurant, Then, we use $k$-means clustering to divide the locations into different regions according to their coordinates as depicted in Figure 1 and use $\mathcal{R}=\left\{r_{j}\right\}_{|\mathcal{R}|}(|\mathcal{R}|$ is the number of regions) to denote the region set. The review set is denoted by $\mathcal{V}=\left\{v_{l, t_{h}}^{u}\right\}_{|\mathcal{V}|}(|\mathcal{V}|$ is the number of reviews), where $v_{l, t_{h}}^{u}$ refers to as the review written by user $u$ when he visited location $l$ at time $t_{h}$. Suppose that all locations have $K$ aspects and we use a $K$-dimensional vector to represent the aspects of a given location $l$, where $a_{t_{h}, k}^{l}$ indicates the users' overall sentiment polarity on aspect $a_{k}$ of location $l$ at $t_{h}$. A higher value of $a_{t_{h}, k}^{l}$ indicates that the sentiment polarity is more positive and vice versa.

To better explain our model, the graphical representation of the static relationships between different factors is shown in Figure 2. A user $u$ decides to visit a location $l$ by considering region $r$ and category $c$. The users who intend to visit the locations of the same category $c$ in the same region $r$ are divided into group $g$. After visiting the location $l$, the user $u$ gives the reviews to show his sentiment $s$ on the aspect $a$ of the location $l$.

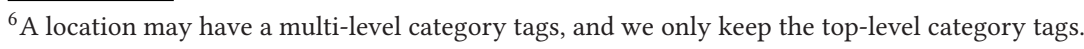




\subsection{User Grouping}

In Liu et al. [19], Wang et al. [27], and Zhao et al. [39], the probability that a user visits a location is affected by the category and region that the location belongs to. We decide to apply a multigroup dividing method to guarantee the recommendation diversity. We partition the locations into $|\mathcal{R}| \times|C|$ groups by considering both region and category factors-for instance, for a location $l \in \mathcal{L}_{b}\left(b=(i-1)^{*}|\mathcal{R}|+j\right)$, it means that $l$ lies in region $r_{i}$ and belongs to category $c_{j}$.

We denote $P_{u, b}$ as the frequency that a user $u$ visits the locations in $\mathcal{L}_{b}$, which represents his interest in $\mathcal{L}_{b}$. Then, a latent factor model (LFM) is applied, and we use user latent matrix $E \in \mathbb{R}^{M \times|\mathcal{U}|}$ and location latent matrix $H \in \mathbb{R}^{M \times(|\mathcal{R}| \times|\mathcal{C}|)}$ to fit the users' interest matrix $P \in \mathbb{R}^{|\mathcal{U}| \times(|\mathcal{R}| \times|\mathcal{C}|)}$, where $M$ is the number of latent factors. Compared with the basic LFM, the probabilistic matrix factorization (PMF) model [24] assigns the users with very few ratings with feature vectors that are close to the average user, so the predicted ratings for those users will be close to the average ratings. In reality, the users prefer to follow the crowd, so we use the PMF model to divide the users into groups according to their latent spaces. Referring to Salakhutdinov and Mnih [24], the latent matrices and the conditional distribution over the observed check-in frequencies follow the normal distribution, and the optimization goal is to minimize the following optimization function $J$ as shown in Equation (1).

$$
J=\arg \min \frac{\left(P-E^{T} H\right)^{2}}{2 \sigma^{2}}+\frac{\left(E^{T} E\right)^{2}}{2 \sigma_{E}^{2}}+\frac{\left(H^{T} H\right)^{2}}{2 \sigma_{H}^{2}}
$$

Then, we perform gradient descent in $E$ and $H$ to minimize the optimization function $J$. After all gradients are calculated, the matrices $E, H$ are calculated. We set a threshold $\gamma$ to partition the users and use $E_{u, m}$ to denote the $m$-th latent factor value of user $u$. The partition rule is that a user $u$ is classified into $m$-th group $g_{m}$ when $E_{u, m} \geq \gamma$. Then, we normalize the probabilities that a user is divided into different groups. Therefore, the users are divided into $M$ groups by jointly considering the category and region factors.

\subsection{Aspect-Based Sentiment Analysis}

To incorporate sentiment into our models, two matrices are generated based on the reviews by using sentiment analysis techniques such as the location sentiment rating matrix and user attention matrix. Table 2 shows an example of a user's real review text on Yelp. Next, we introduce how to mine users' opinions from their reviews.

Aspect extracting has been well studied by existing work [15, 21]. In our article, we only focus on common representative aspects and use a basic LDA model [2] to acquire the word distributions of different topics. Those words expressing the same facet are automatically grouped together under the same topic. An aspect can be expressed by a set of words characterizing a topic in the given domain, which can be features of items or attributes of services. We manually select the appropriate words under the corresponding topics as aspect words. Table 3 shows some aspects and the corresponding aspect words. For example, "value," "price," and "worth" are all under the same topic, which are chosen as the aspect words to express the aspect "price." $W_{k}$ denotes the set of aspect words mapping to aspect $a_{k}$.

Next, we construct a sentiment lexicon $\mathcal{S}$ from user reviews by applying the methods in $\mathrm{Lu}$ et al. [19] and the general-purpose sentiment lexicon provided to construct $\mathcal{S}$ is SentiWordNet 3.0. ${ }^{7}$ Then, we analyze users' opinions by following the work of Jo and Oh [13] and Zhao et al. [40] and first extract the aspect-opinion entries (i.e., $(w, o))$ from the users' reviews, where $w \in \mathcal{W}_{k}$ is an aspect word to express aspect $a_{k}$ and $o$ is an opinion word. For instance, in the example shown

$\overline{{ }^{7} \text { http://sentiwordnet.isti.cnr.it/. }}$ 
Table 2. Example of a User's Review

UserId: -50XWnmQGqBgEI-9ANvLlg
LocationId: DYuOxkW4DtlJsTHdxdXSlg
Star: 5
Date: $2014 / 6 / 5$
Text: The portions are huge! Ambience is nice and casual. Had a Spicy Mango Mojito
breaded with Shiracha Mayo drizzled on top and a small bowl on the side. The mayo
was so good we used it on everything after this course! Then we had the Jerk Chicken
Pasta with a side Caesars salad and the Island Hopper appetizer as a meal. The pasta
was loaded with asparagus and mushroom with some chicken all cooked well. The light
portion was huge, so no need for the regular portion. The side salad was fresh and tasty,
but absolutely too big. The Hopper was tasty with great coconut shrimp, huge crispy
jerk wings, big onion ring and conch fritters. WAY too much food!! Everything was good,
but the 3 sauces on the platter can't hold a candle to the Shiracha mayo! Would definitely
return here, but wait for happy hour to get $1 / 2$ off appetizers and drinks.

Table 3. Example of Aspects

\begin{tabular}{c|l}
\hline Aspect & \multicolumn{1}{c}{ Aspect Words } \\
\hline Food & Food, taste, dish, flavor, snack, appetizer... \\
\hline Service & Service, waitress, waiter, service, assistance, assist... \\
\hline Traffic & Traffic, park, parking space, walk, station... \\
\hline Environment & Environment, atmosphere, surrounding... \\
\hline
\end{tabular}

in Table 2 ("The portions are huge! Ambience is nice and casual."), the entries (portion, huge) and (environment, nice) could be extracted. Because "portion" and "Ambience" are characterizing the aspects "food" and "environment." Through consulting the lexicon $\mathcal{S}$, we can acquire the sentiment polarity of entry $(w, o)$ according to the opinion word $o$. In particular, the polarity value +1 represents that the entry is positive, -1 for negative, and 0 for neutral. Then, negation words detection is conducted to check whether the sentiment of each entry is reversed. In this example, we acquire the aspect-sentiment entry (food, huge, +1 ) and (environment, nice, +1 ).

By summing up the polarity values of all related entries extracted from the reviews that published before $t_{n}$ on location $l$, the average aspect-sentiment score is calculated by $s_{t_{n}, k}^{l}=$ $\frac{1}{N_{k}^{l}} \sum_{w \in \mathcal{W}_{k}}(w, o)$, which reflects the users' overall sentiment orientation on the $k$-th aspect of $l$ at $t_{n}$, where $N_{k}^{l}$ is the number of corresponding entries on $a_{k}$. In the location sentiment rating matrix, we use each element $y_{t_{n}, k}^{l}$ to denote the composite score of location $l$ on aspect $a_{k}$ at time $t_{n}$ which is defined as follows:

$$
y_{t_{n}, k}^{l}=\frac{z_{t_{n}}^{l}}{1+e^{-\beta s_{t_{n}, k}^{l}}} .
$$

$z_{t_{n}}^{l}$ is the average rating of the location $l$ in $\mathcal{T}_{n}$. Our definition considers the quality of location on aspect level through analyzing users' reviewers. The composite score contains two parts: the average rating $z_{t_{n}}^{l}$ and the user sentiment score $\frac{1}{1+e^{-\beta s_{t_{n}, k}^{l}}}$. Similarly, we use a $K$-dimension vector to describe a user's attention degree on $K$ aspects. $f_{k}^{u}$ is used to count the times that $u$ mentions $a_{k}$ in his reviews (i.e., the number of aspect-sentiment pairs about $a_{k}$ ). In the user attention matrix, 


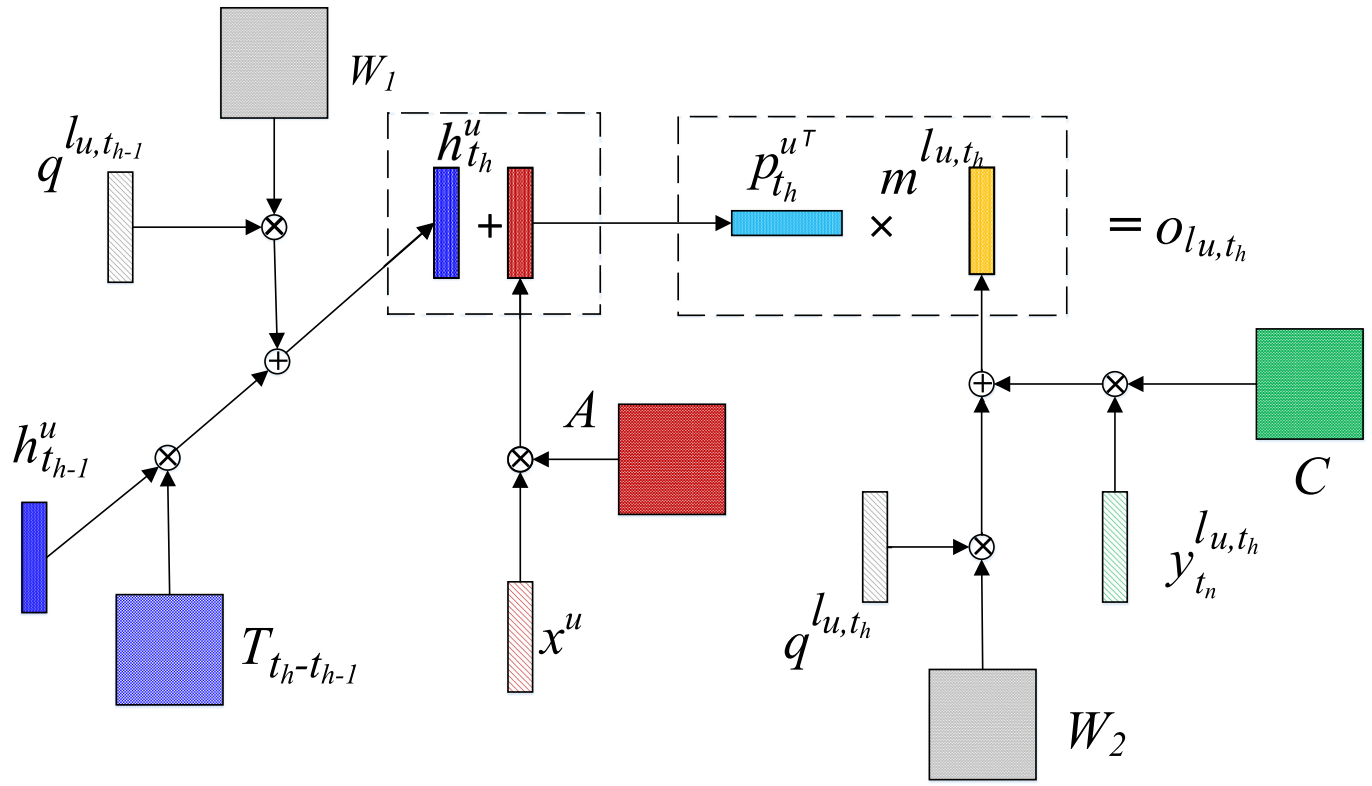

Fig. 3. Framework of GTSAR-RNN.

we follow the setting in Zhang et al. [36] that uses $x_{k}^{u}$ to denote the global attention of user $u$ on aspect $a_{k}$ in $\mathcal{T}_{n}$ as follows:

$$
x_{k}^{u}=\left\{\begin{array}{ll}
1 & \text { if } f_{k}^{u} \text { is } 0 \\
1+(N-1)\left(\frac{2}{1+e^{-f_{k}^{u}}}-1\right) & \text { if } f_{k}^{u} \neq 0
\end{array} .\right.
$$

The choice of $N$ is 5 in many real-world five star-based reviewing systems, such as Amazon and Yelp. If an aspect is not mentioned by the user, we still assign a private value of 1 to it. We further normalize the weight vector $x^{u}$, which makes $\sum_{1}^{K} x_{k}^{u}=1$.

\subsection{Neural Architecture}

To better represent each location, we decide to apply the embedding vector instead of one-hot coding. The neural architecture of our model is depicted in Figure 3. Considering the dynamic

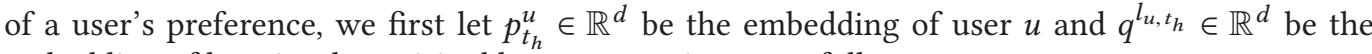
embedding of location $l_{u, t_{h}}$ visited by user $u$ at time $t_{h}$ as follows:

$$
p_{t_{h}}^{u}=\left(h_{t_{h}}^{u}+A x^{u}\right)
$$

where $A \in \mathbb{R}^{d \times d}$ is the attention transition matrix that captures a user's aspect-based attention extracted from his reviews. We use $h_{t_{h}}^{u}$ to denote the visit embedding of user $u$ at $t_{h}$ that captures his dynamic interest as follows:

$$
h_{t_{h}}^{u}=f\left(W_{1} q^{l_{u, t_{h-1}}}+T_{t_{h}-t_{h-1}} h_{t_{h-1}}^{u}\right),
$$

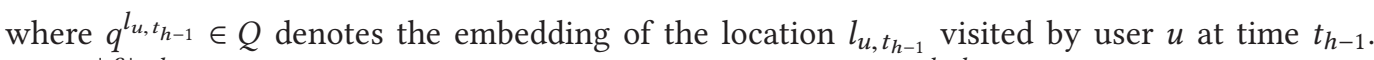
$Q \in \mathbb{R}^{|\mathcal{L}| \times d}$ is the embedding matrix of all locations. $T_{t_{h}-t_{h-1}} \in \mathbb{R}^{d \times d}$ is the time transition matrix that captures temporal contexts. Relu is chosen as the activation function $f(x)$. For simplicity, we 
directly use the definition in Zhang et al. [37]:

$$
T(t)= \begin{cases}\frac{\pi-t}{\pi} T_{0}+\frac{t}{\pi} T_{\pi} & t<\pi \\ T_{\pi} & t>\pi\end{cases}
$$

where $T_{0}, T_{\pi}$ are two transition matrices and $t$ is a time interval. When time interval $t$ goes beyond the threshold, only $T_{\pi}$ is adopted in calculation; otherwise, $T_{0}, T_{\pi}$ are both in charge of calculation. Later in Equation (18), the derivation of $J$ with respect to $T$ is replaced by the derivations with respect to $T_{0}$ and $T_{\pi}$.

Second, we define the representation of location $l_{u, t_{h}}$ visited by $u$ at time $t_{h}$ consisting of two parts: basic location embedding $q^{l_{u, t_{h}}}$ and sentiment embedding $y_{t_{n}}^{l_{u, t_{h}}}$. It is noted that $q^{l_{u, t_{h}}}$ is the basic embedding of location $l_{u, t_{h}}$ that does not consider the effect of the review sentiment. Similar to the user, the attraction of location is also time aware, which can be reflected by the users' reviews. So we use $m^{l_{u, t_{h}}}$ to represent the combined embedding of location $l_{u, t_{h}}$ at $t_{h}$ :

$$
m^{l_{u, t_{h}}}=W_{2} q^{l_{u, t_{h}}+C y_{t_{n}}^{l_{u, t_{h}}} .}
$$

$C \in \mathbb{R}^{d \times K}$ is the sentiment transition matrix that maps $K$-dimension explicit sentiment score into $d$-dimension latent embedding. $y_{t_{n}}^{l_{u, t_{h}}} \in \mathbb{R}^{K \times 1}$ captures the users' opinions on the specified $K$ aspects of location $l_{u, t_{h}}$. It is inconvenient to calculate the users' average sentiment on $l_{u, t_{h}}$ at time $t_{h}$, so we use $y_{t_{n}, t_{h}}^{l_{u}}$ to replace it, where $t_{h} \in \mathcal{T}_{n}$.

Finally, the prediction score of GTSAR-RNN can be yielded via calculating the inner product of user and location representations. $o_{l_{u, t_{h}}}$ is used to denote the probability that the user $u$ visits the location $l_{u, t_{h}}$ at time $t_{h}$, which can be calculated as

$$
o_{l_{u, t_{h}}}=\left(p_{t_{h}}^{u}\right)^{T} m^{l_{u, t_{h}}} .
$$

To train our model, we adopt Bayesian personalized ranking (BPR) [22] and back-propagation through time (BPTT)[9]. BPR is a pairwise ranking method for the implicit factor based on the basic assumption that a user prefers a selected location rather than a negative one. Incorporating the negative log likelihood, we can solve the following objective function equivalently:

$$
J=\sum \ln \left(1+e^{-\left(o_{l_{u, t_{h}}}-o_{l_{u, t_{h}}^{\prime}}\right)}\right)+\frac{\lambda}{2}\|\Theta\|^{2},
$$

where $\Theta=\left\{Q ; W_{1} ; W_{2} ; T ; A ; C\right\}$ denotes all parameters to be estimated and $\lambda$ is a parameter to control the power of regularization. The derivations of $J$ with respect to the parameters can be calculated as

$$
\begin{aligned}
& \frac{\partial J}{\partial p_{t_{h}}^{u}}=\sum \frac{\left(m^{l_{u, t_{h}}^{\prime}}-m^{\left.l_{u, t_{h}}\right)} e^{-\left(o_{l_{u, t_{h}}}-o_{l_{u, t_{h}}^{\prime}}\right)}\right.}{1+e^{-\left(o_{l_{u, t_{h}}}-o_{l_{u, t_{h}}^{\prime}}\right)}}+\lambda p_{t_{h}}^{u}, \\
& \frac{\partial J}{\partial m^{l_{u, t_{h}}}}=-\sum \frac{p_{t_{h}}^{u} e^{-\left(o_{l_{u, t_{h}}}-o_{l_{u, t_{h}}^{\prime}}\right)}}{1+e^{-\left(o_{l_{u, t_{h}}}-o_{l_{u, t_{h}}^{\prime}}\right)}}+\lambda m^{l_{u, t_{h}}}, \\
& \frac{\partial J}{\partial m^{l_{u, t_{h}}^{\prime}}}=\sum \frac{p_{t_{h}}^{u} e^{-\left(o_{l_{u, t_{h}}}-o_{l_{u, t_{h}}^{\prime}}\right)}}{1+e^{-\left(o_{l_{u, t_{h}}}-o_{l_{u, t_{h}}^{\prime}}\right)}}+\lambda m^{l_{u, t_{h}}^{\prime}},
\end{aligned}
$$

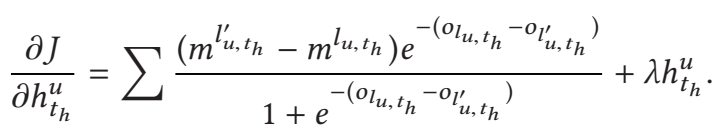


$m^{l_{u, t_{h}}^{\prime}} \in Q$ is the embedding of negative sample-for instance, a location that is not visited by user $u$ at time $t_{h}$. Moreover, parameters in our model can be further learned with the BRTT algorithm. Given the derivation $\frac{\partial J}{\partial h_{t_{h}}^{u}}$, the corresponding gradients of other parameters can be calculated as

$$
\begin{aligned}
& \frac{\partial J}{\partial A}=\left(I \otimes \frac{\partial J}{\partial p_{t_{h}}^{u}}\right)\left(x^{u}\right)^{T}, \\
& \frac{\partial J}{\partial C}=\left(I \otimes\left(\frac{\partial J}{\partial m^{l_{u, t_{h}}}}-\frac{\partial J}{\partial m^{l_{u, t_{h}}^{\prime}}}\right)\right)\left(y_{t_{n}}^{l_{u, t_{h}}}\right)^{T}, \\
& \frac{\partial J}{\partial W_{1}}=\left(f^{\prime}(.) \otimes \frac{\partial J}{\partial h_{t_{h}}^{u}}\right)\left(q^{\left.l_{u, t_{h-1}}\right)^{T}},\right. \\
& \frac{\partial J}{\partial W_{2}}=\left(I \otimes\left(\frac{\partial J}{\partial m^{l_{u, t_{h}}}}-\frac{\partial J}{\partial m^{l_{u, t_{h}}^{\prime}}}\right)\right)\left(q^{l_{u, t_{h}}}\right)^{T}, \\
& \frac{\partial J}{\partial T}=\left(f^{\prime}(.) \otimes \frac{\partial J}{\partial h_{t_{h}}^{u}}\right)\left(h_{t_{h-1}}^{u}\right)^{T}, \\
& \frac{\partial J}{\partial q^{l_{u, t_{h-1}}}}=\left(f^{\prime}(.) \otimes \frac{\partial J}{\partial h_{t_{h}}^{u}}\right)\left(W_{1}\right)^{T}, \\
& \frac{\partial J}{\partial q^{l_{u, t_{h}}}}=\left(I \otimes\left(\frac{\partial J}{\partial m^{l_{u, t_{h}}}}-\frac{\partial J}{\partial m^{l_{u, t_{h}}^{\prime}}}\right)\right)\left(W_{2}\right)^{T}, \\
& \frac{\partial J}{\partial m^{l_{u, t_{h}}^{\prime}}}=\left(I \otimes\left(\frac{\partial J}{\partial m^{l_{u, t_{h}}}}-\frac{\partial J}{\partial m^{l_{u, t_{h}}^{\prime}}}\right)\right)\left(W_{2}\right)^{T} .
\end{aligned}
$$

Now, we can employ gradient descent to estimate the model parameters, after all gradients are calculated. This process can be repeated iteratively until the convergence is achieved.

For a user $u \in g_{i}$, we input his check-in sequence and the embeddings of candidate locations into the corresponding networks that have been trained. Here, we can get the visit probability $p\left(l \mid u, g_{i}\right)$ for each location $l$. By merging his predicting results of different groups, the final probability that $u$ visits $l$ can be calculated as follows:

$$
\operatorname{Pr}(l \mid u)=\sum_{g_{i} \in \mathcal{G}_{u}} \operatorname{Pr}\left(l \mid g_{i}, u\right) \operatorname{Pr}\left(g_{i} \mid u\right)
$$

where $\mathcal{G}_{u}$ is the group set that user $u$ belongs to and a user can be divided into several groups by comprehensively considering the category tags and the geographical coordinates of the locations that the users have visited. $\operatorname{Pr}\left(g_{i} \mid u\right)$ is the normalized value of $E_{u, i}$ calculated by $\frac{E_{u, i}}{\sum_{E_{u, i^{\prime}}>\gamma} E_{u, i^{\prime}}}$. Then, we rank the probabilities of different locations to be visited and select the top- $k$ results for recommendation.

\section{EXPERIMENT}

In this section, we conduct empirical experiments to demonstrate the effectiveness of GTSARRNN on POI recommendation. We first introduce the datasets, baseline methods, and evaluation metrics of our experiments. Then, we compare the proposed GTSAR-RNN with the state-of-the-art baseline methods. Finally, we show the process of parameter tuning and synthesis analysis. 
Table 4. Data Statistics

\begin{tabular}{|c|c|c|c|}
\hline & Las Vegas & Phoenix & Scottsdale \\
\hline Locations & 22,014 & 13,844 & 6,504 \\
\hline Users & 22,870 & 17,899 & 14,396 \\
\hline Reviews & 526,472 & 225,342 & 117,368 \\
\hline Reviews per user & 23.020 & 12.590 & 8.153 \\
\hline Reviews per location & 23.915 & 16.277 & 18.046 \\
\hline
\end{tabular}

Table 5. Category Set

Restaurants, Shopping, Food, Beauty\&Spas, Nightlife, Bars, Fashion
Health\&Medical, Arts\&Entertainment, Automotive, Event Planning\&Services
Active Life, Hotels\&Travel, Home Services

\subsection{Experimental Settings}

We evaluate different methods based on real datasets from Yelp, which contains 1,183,362 users, 156,640 locations, and 4,736,898 reviews from 2007 to 2017. The average number of reviews per user is 4.002 , and the average number of reviews per location is 30.240 . We classify these data according to the cities and use the three largest datasets for conducting experiments. The statistics of three datasets are shown in Table 4. We remove the users whose visiting records are less than 10. For each dataset, we take the recent $20 \%$ check-in data of each user for testing and use the remaining $80 \%$ data for training our model. To test the effectiveness of a recommendation result, we make a judgment by checking whether the user visits the recommended location and gives a more than a three-star rating.

Before training our model, aspect-based sentiment analysis methods are applied in extracting users' sentiment on different locations. We finally select 12 aspects that are mostly mentioned by the users in our experiments. Specifically, we analyze the hierarchical structure of different categories and get 14 categories at the top as depicted in Table 5. Each location must belong to at least one of these categories.

\subsection{Evaluation Metrics and Baselines}

In this work, we employ two evaluation metrics-Precision@k and NDCG@k-that popularly are used in ranking tasks. The evaluation score for our experiment is computed based on the position where the next selected location appears in the ranked list. We report Precision@k and NDCG@k with $\mathrm{k}=5,10$, and 15, respectively, in our experiments.

We compare our approaches against two naive baselines and four improved methods proposed in different literature. All comparison systems are subject to the same filtering and preprocessing procedures as our own algorithm. One basic baseline is the user-based CF model, which predicts a user's preferences by considering the preferences of other similar users. To measure the similarity between the users, we use the Pearson correlation coefficient. The other one is the RNN, which treats a user's check-in data as a sequence for prediction. All baselines are presented as follows,

- CF: This user-based CF model is a basic model that utilizes user similarity for recommendation.

- PMF [24]: PMF is a probabilistic linear model with a prior, which first models users' rating behaviors following the Gaussian distribution. 
Table 6. Parameters

\begin{tabular}{|c||c|}
\hline Parameter & Values \\
\hline Layer size & 6 \\
\hline Learning rate & $0.005 \sim 0.01$ \\
\hline Embedding dimensionality $d$ & $10 \sim 40$ \\
\hline Region number $|\mathcal{R}|$ & $8 \sim 17$ \\
\hline Group number $M$ & $10 \sim 25$ \\
\hline Threshold parameter $\lambda$ & $0 \sim 1$ \\
\hline Sentiment parameter $\beta$ & $0 \sim 5$ \\
\hline
\end{tabular}

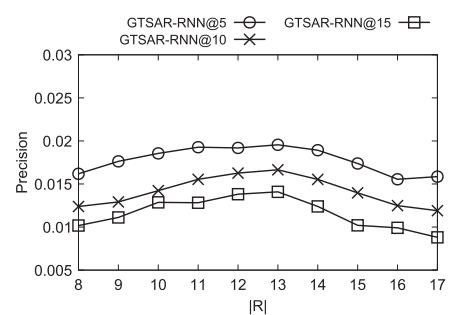

(a)Las Vegas

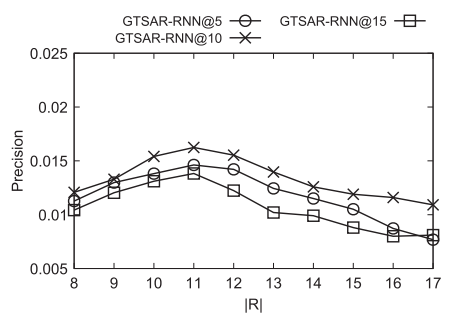

(b)Phonix

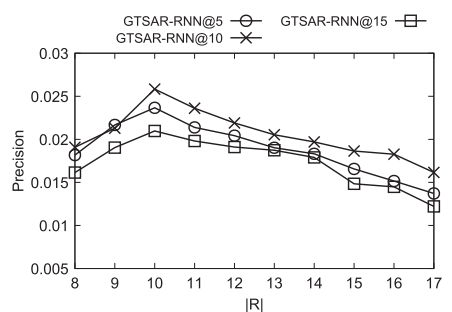

(c)Scottsdale

Fig. 4. Varying $|\mathcal{R}|$.

- GCF [18]: GCF is a CF model incorporating geographical influence. However, without considering the content of the reviews, GCF cannot model a user's preferences on an aspect level.

- FPMC-LR [6]: The MC model is a classical sequential model and can be used as a sequential baseline method. FPMC-LR exploits the personalized MC in the check-in sequence and also takes into account users' movement constraint (i.e., a user moves around a localized region).

- Rank-GeoFM [14]: Rank-GeoFM addresses the data scarcity problem and incorporates geographical influence. The proposed model learns the factorization by ranking the POIs correctly considering that the check-in frequency characterizes users' visiting preferences.

- NEXT [37]: NEXT is an unified framework to learn the hidden intent regarding a user's next move by incorporating different factors in a unified manner, which is able to incorporate temporal context, sequential relations, geographical influence, and auxiliary metadata information in an integrated architecture.

- RNN [35]: RNN can be used for temporal prediction directly, which has been successfully applied in word embedding and ad click prediction. It inputs the user's check-in data according to his visiting order and outputs the next location for recommendation.

- ST-RNN [17]: This is an extended RNN model that models the spatial temporary contexts by means of a latent model. To capture time interval and geographical distance information, STRNN replaces the single transition matrix in the RNN with time-specific transition matrices and distance-specific transition matrices.

\subsection{Parameters}

In this section, we study the impact of different parameters on the performance of our model. Table 6 gives the parameters and value ranges. In GTSAR-RNN, the impact of geographical factors on POI recommendation is controlled by the region number $|\mathcal{R}|$, which is shown in Figure 4 . On 


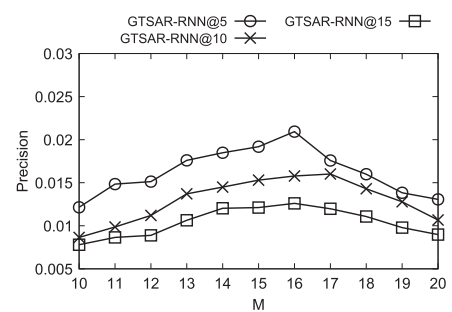

(a)Las Vegas

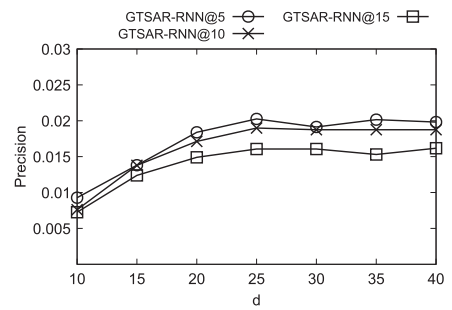

(a)Las Vegas

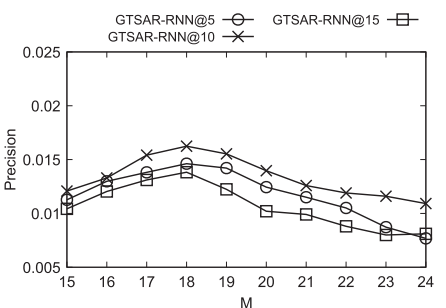

(b)Phonix

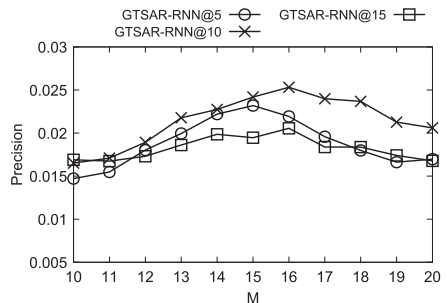

(c)Scottsdale

Fig. 5. Varying $M$.

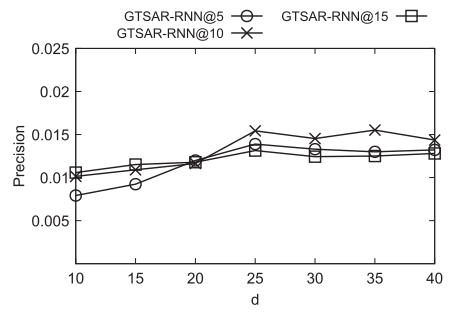

(b)Phonix

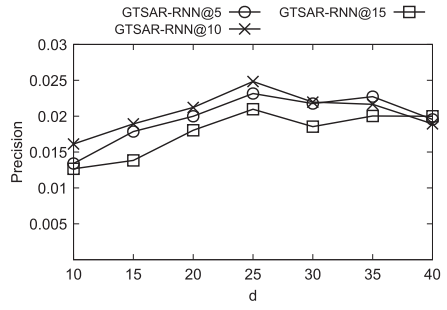

(c)Scottsdale

Fig. 6. Varying $d$.

the Las Vegas dataset, the best performance of GTSAR-RNN is obtained with $|\mathcal{R}|=13$. The most suitable region number on Phoenix and Scottsdale are 11 and 10, respectively, which indicates that the bigger dataset requires more region divisions.

Specifically, the grouping result has a great influence on the final performance, which are controlled by the group number $M$. Figure 5 shows the precision of GTSAR-RNN with respect to different $M$ ranging from 10 to 25 with an increment of 1 on different datasets. It is worth noting that the best group number of the Las Vegas dataset is smaller than that of the Phoenix dataset. In general, the larger dataset requires a larger group number. A reasonable explanation is that Las Vegas is a famous entertainment city, and most people go there for fun and entertainment locations in specific areas. Therefore, these people tend to have similar preferences, which is why they are inclined to be divided into smaller numbers of groups.

Figure 6 shows the precision of GTSAR-RNN with respect to different $d$ ranging from 10 to 40 on different datasets. On the Las Vegas dataset, the precision of GTSAR-RNN continues to increase with increasing $d$ and remains stable when $d$ goes beyond 30. The best performances on the Phoenix and Scottsdale datasets are both acquired when $d=25$.

Figure 7 (a) shows the precision of GTSAR-RNN (setting $|\mathcal{R}|=13,|C|=14$ ) with respect to different $\gamma$ ranging from 0 to 0.3 on the Las Vegas dataset. The most suitable value for $\gamma$ and $M$ is 16 when the other parameters are fixed. $\beta$ is a parameter to control the scale of the sentiment score, and the most suitable $\beta$ value is 1.1 as depicted in Figure 7(b). We use the same value settings for $\gamma$ and $\beta$ on the other two datasets, which also gain a good effect on the other datasets.

We test the performance of GTSAR-RNN with different grouping methods, and the comparison results are shown in Figure 8. As mentioned earlier, PMF represents probabilistic matrix factorization and LFM [7] represents the latent factor model. $k$-Means clustering and spectrum clustering are two popular methods, but they can only partition each user into one group. The comparison 


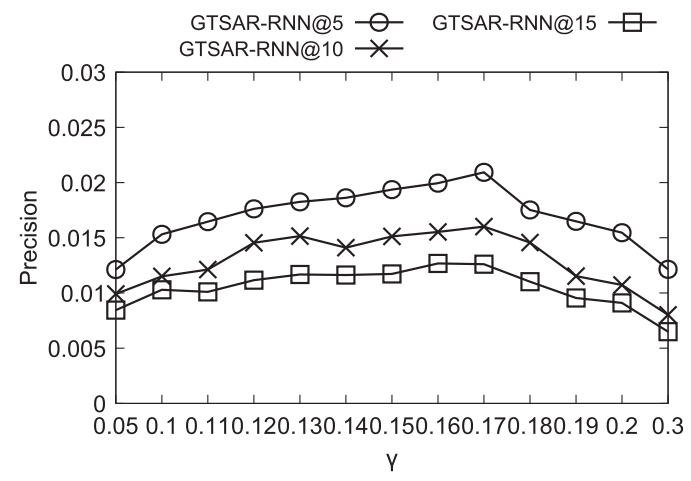

(a)varying $\gamma$

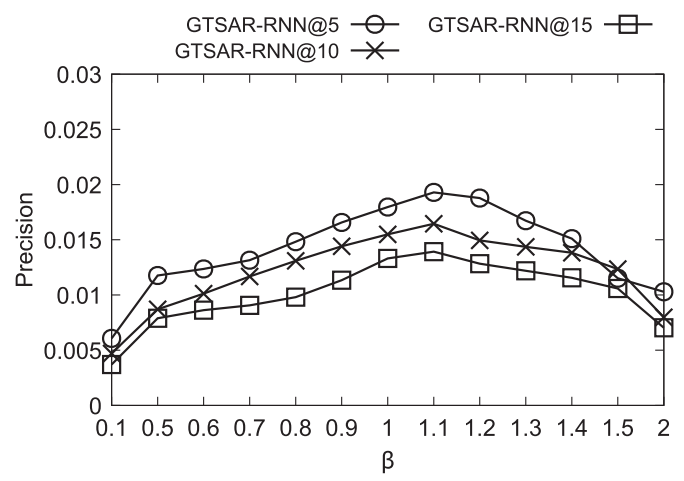

(b)varying $\beta$

Fig. 7. Varying $\gamma$ and $\beta$.

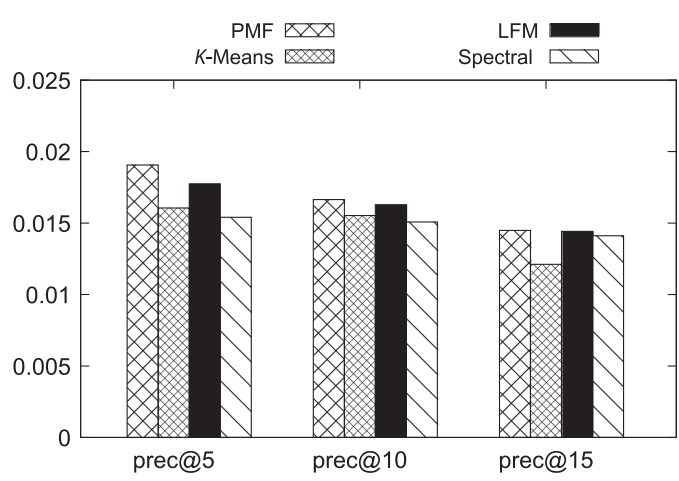

Fig. 8. Comparison of different grouping methods.

result proves that multi-grouping methods perform better than single-grouping methods and PMF acquires the best performance.

\subsection{Comprehensive Analysis}

The performance comparisons on the datasets evaluated by precision and NDCG are illustrated in Figure 9. CF and GCF both take the collaborative information into consideration but cannot model the temporal information. By taking geographical effect into consideration, GCF and Rank-GeoFM both improve the recommending results compared with the traditional CF model. FPMC-LR is a kind of sequential prediction method that captures the user's serial behavior, and it outperforms the GCF, CF, and RNN, obviously. As an enhanced prediction method, ST-RNN achieves further improvement compared with FPMC-LR by incorporating spatial-temporal factors. We observe that NEXT and ST-RNN perform better than other methods. The probable reason may be that these two methods synthetically consider the time influence and geographical influence. Compared with the preceding methods, we consider more influence factors and the experimental results demonstrate the effectiveness of our proposed method.

On the Scottsdale dataset, our method shows the largest improvement. Compared with the second best method ST-RNN, the improvements of GTSAR-RNN are 16.64\% on prec@5, 17.31\% on prec@10, and 16.20\% on prec@15, respectively. Meanwhile, the improvements are 11.48\% on prec@5, 18.09\% on prec@10, and 16.46\% on prec@15 on the Las Vegas dataset. On the Phoenix 


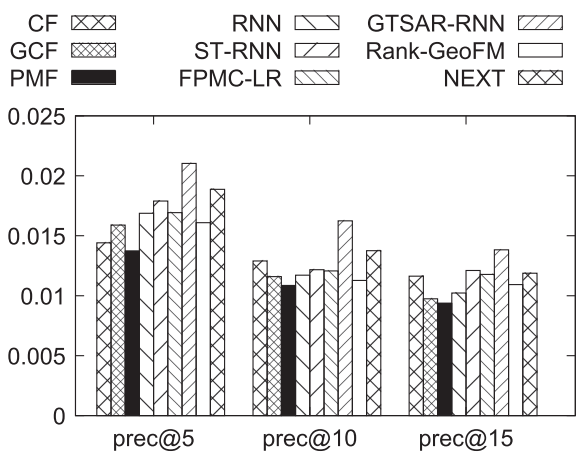

(a) pre@Las Vegas

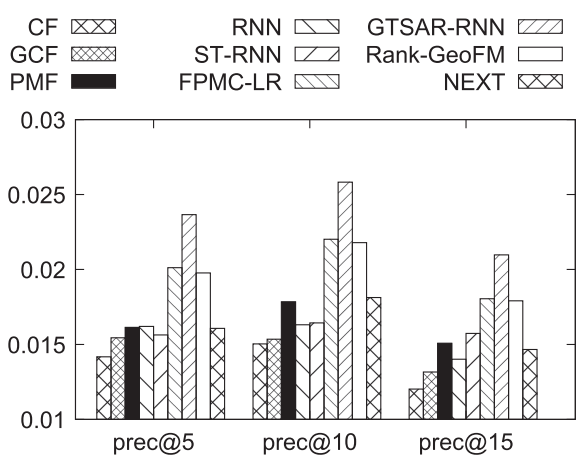

(c) pre@Scottsdale

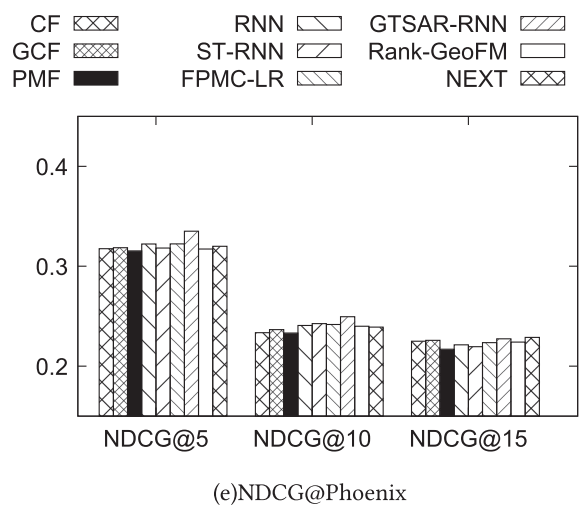

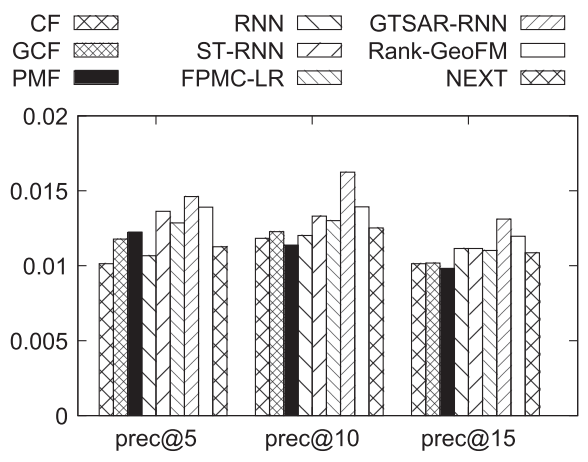

(b) pre@Phoenix

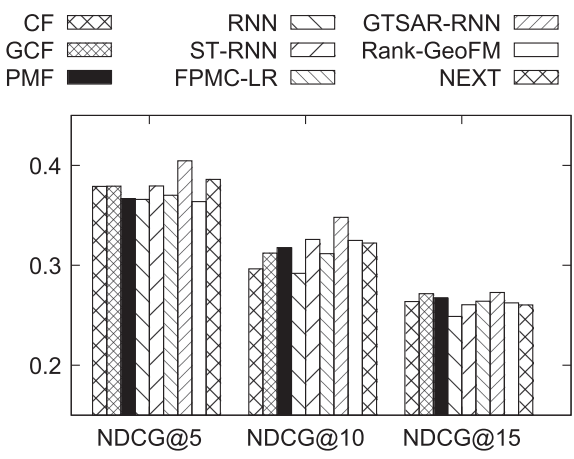

(d)NDCG@Las Vegas
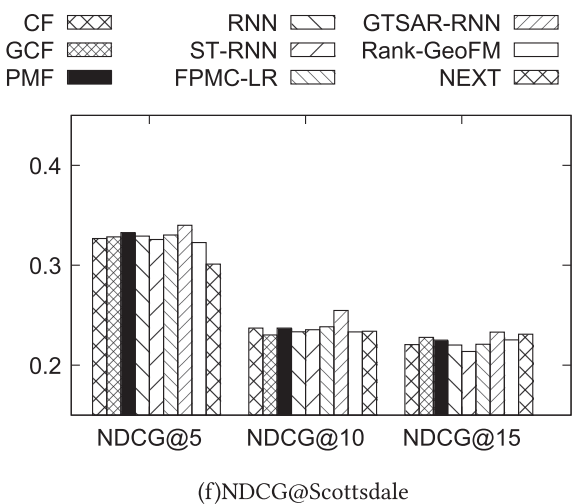

Fig. 9. Experiment comparisons

dataset, the ranking 2 method is NEXT and the improvements of GTSAR-RNN are 5.13\% on prec@5, 16.58\% on prec@10, and 9.58\% on prec@15, respectively.

Similar to precision, there also exist some improvements in various degrees on NDCG. Compared with the second best method ST-RNN, GTSAR-RNN has improvements of $2.96 \%$ on NDCG@5,6.84\% on NDCG@10, and 5.54\% on NDCG@15 on the Scottsdale dataset and 4.84\% on NDCG@5, 7.99\% on NDCG@10,4.76\% on NDCG@15 on the Las Vegas dataset. On the Phoenix dataset, the improvements of GTSAR-RNN are 5.61\% on NDCG@5, 3.94\% on NDCG@10, and 1.43\% on NDCG@15 compared to the second best method NEXT. 


\section{CONCLUSION}

In this article, we propose the group-based neural network POI recommendation model GTSARRNN. Different from traditional sequence predicting methods such as RNN and MC, GTSAR-RNN first integrates the user's sentiment information with spatial-temporal contexts. GTSAR-RNN predict the user's visit intent by calculating the inner product of user embedding and location embedding. Under this framework, we can incorporate different contextual factors into a unified framework for POI recommendation. The experimental results on real datasets show that our GTSAR-RNN outperforms the state-of-the-art methods.

As a novel model that incorporates sentiment information into a neural network model for recommendation, it still leaves much to be desired. Instead of modeling the spatial-temporal context and sentiment independently in this article, we will further study the interaction between temporal-spatial information and sentiment orientation. Moreover, it lacks enough explication for the recommendation results. To improve the explication, it is worthwhile to further study the modeling of these utilities (user trust, satisfaction, etc.) and generating explanations automatically in the future.

\section{REFERENCES}

[1] Dzmitry Bahdanau, Kyunghyun Cho, and Yoshua Bengio. 2014. Neural machine translation by jointly learning to align and translate. arXiv:1409.0473.

[2] David M. Blei, Andrew Y. Ng, and Michael I. Jordan. 2003. Latent Dirichlet allocation. fournal of Machine Learning Research 3 (2003), 993-1022. http://www.jmlr.org/papers/v3/blei03a.html.

[3] John S. Breese, David Heckerman, and Carl Myers Kadie. 1998. Empirical analysis of predictive algorithms for collaborative filtering. In Proceedings of the 14th Conference on Uncertainty in Artificial Intelligence (UAI'98). 43-52. https://dslpitt.org/uai/displayArticleDetails.jsp?mmnu=1\&smnu=2\&article_id=231\&proceeding_id=14.

[4] Xin Cao, Gao Cong, and Christian S. Jensen. 2010. Mining significant semantic locations from GPS data. Proceedings of the VLDB Endowment 3, 1 (2010), 1009-1020. DOI : https://doi.org/10.14778/1920841.1920968

[5] Chen Cheng, Haiqin Yang, Irwin King, and Michael R. Lyu. 2016. A unified point-of-interest recommendation framework in location-based social networks. ACM Transactions on Intelligent Systems and Technology 8, 1 (2016), Article 10, 21 pages. DOI : https://doi.org/10.1145/2901299

[6] Chen Cheng, Haiqin Yang, Michael R. Lyu, and Irwin King. 2013. Where you like to go next: Successive point-ofinterest recommendation. In Proceedings of the 23rd International foint Conference on Artificial Intelligence (IFCAI'13). 2605-2611. http://www.aaai.org/ocs/index.php/IJCAI/IJCAI13/paper/view/6633.

[7] Jian Cheng, Ting Yuan, Jinqiao Wang, and Hanqing Lu. 2014. Group latent factor model for recommendation with multiple user behaviors. In Proceedings of the 37th International ACM SIGIR Conference on Research and Development in Information Retrieval (SIGIR'14). 995-998. DOI : https://doi.org/10.1145/2600428.2609493

[8] Paul Covington, Jay Adams, and Emre Sargin. 2016. Deep neural networks for YouTube recommendations. In Proceedings of the 10th ACM Conference on Recommender Systems. 191-198. DOI : https://doi.org/10.1145/2959100.2959190

[9] G. E. Hinton, D. E. Rumelhart, and R. J. Williams. 1986. Learning representations by back-propagating errors. Nature 323 (1986), 533-536.

[10] Shanshan Feng, Xutao Li, Yifeng Zeng, Gao Cong, Yeow Meng Chee, and Quan Yuan. 2015. Personalized ranking metric embedding for next new POI recommendation. In Proceedings of the 24th International Conference on Artificial Intelligence (IFCAI'15). 2069-2075. http://ijcai.org/Abstract/15/293.

[11] Lei Guo, Jun Ma, Zhumin Chen, and Huan Zhong. 2015. Learning to recommend with social contextual information from implicit feedback. Soft Computing 19, 5 (2015), 1351-1362. DOI : https://doi.org/10.1007/s00500-014-1347-0

[12] Yohan Jo and Alice H. Oh. 2011. Aspect and sentiment unification model for online review analysis. In Proceedings of the 4th ACM International Conference on Web Search and Data Mining (WSDM'11). 815-824. DOI: https://doi.org/10. $1145 / 1935826.1935932$

[13] Junghoon Lee, In-Hye Shin, and Gyung-Leen Park. 2008. Analysis of the passenger pick-up pattern for taxi location recommendation. In Proceedings of the 2008 4th International Conference on Networked Computing and Advanced Information Management. 199-204. DOI: https://doi.org/10.1109/NCM.2008.24

[14] Xutao Li, Gao Cong, Xiaoli Li, Tuan-Anh Nguyen Pham, and Shonali Krishnaswamy. 2015. Rank-GeoFM: A ranking based geographical factorization method for point of interest recommendation. In Proceedings of the 38th International ACM SIGIR Conference on Research and Development in Information Retrieval (SIGIR'15). 433-442. https://doi.org/10. $1145 / 2766462.2767722$ 
[15] Bing Liu. 2012. Sentiment Analysis and Opinion Mining. Morgan \& Claypool. DOI:https://doi.org/10.2200/ S00416ED1V01Y201204HLT016

[16] Kuan Liu, Xing Shi, Anoop Kumar, Linhong Zhu, and Prem Natarajan. 2016. Temporal learning and sequence modeling for a job recommender system. In Proceedings of the Recommender Systems Challenge (RecSys Challenge'16). Article 7, 4 pages. DOI: https://doi.org/10.1145/2987538.2987540

[17] Qiang Liu, Shu Wu, Liang Wang, and Tieniu Tan. 2016. Predicting the next location: A recurrent model with spatial and temporal contexts. In Proceedings of the 30th AAAI Conference on Artificial Intelligence (AAAI'16). 194-200. http://www.aaai.org/ocs/index.php/AAAI/AAAI16/paper/view/11900.

[18] Yong Liu, Wei Wei, Aixin Sun, and Chunyan Miao. 2014. Exploiting geographical neighborhood characteristics for location recommendation. In Proceedings of the 23rd ACM International Conference on Information and Knowledge Management (CIKM'14). 739-748. DOI : https://doi.org/10.1145/2661829.2662002

[19] Yue Lu, Malú Castellanos, Umeshwar Dayal, and ChengXiang Zhai. 2011. Automatic construction of a context-aware sentiment lexicon: An optimization approach. In Proceedings of the 20th International Conference on World Wide Web (WWW'11). 347-356. DOI : https://doi.org/10.1145/1963405.1963456

[20] Anastasios Noulas, Salvatore Scellato, Neal Lathia, and Cecilia Mascolo. 2012. A random walk around the city: New venue recommendation in location-based social networks. In Proceedings of the 2012 International Conference on Privacy, Security, Risk, and Trust and the 2012 International Conference on Social Computing. 144-153. DOI: https://doi.org/10.1109/SocialCom-PASSAT.2012.70

[21] Guang Qiu, Bing Liu, Jiajun Bu, and Chun Chen. 2011. Opinion word expansion and target extraction through double propagation. Computational Linguistics 37, 1 (2011), 9-27. DOI : https://doi.org/10.1162/coli_a_00034

[22] Steffen Rendle, Christoph Freudenthaler, Zeno Gantner, and Lars Schmidt-Thieme. 2009. BPR: Bayesian personalized ranking from implicit feedback. In Proceedings of the 25th Conference on Uncertainty in Artificial Intelligence (UAI'09). 452-461. https://dslpitt.org/uai/displayArticleDetails.jsp?mmnu=1\&smnu=2\&article_id=1630\&proceeding_id=25.

[23] Steffen Rendle, Christoph Freudenthaler, and Lars Schmidt-Thieme. 2010. Factorizing personalized Markov chains for next-basket recommendation. In Proceedings of the 19th International Conference on World Wide Web (WWW'10). 811-820. DOI : https://doi.org/10.1145/1772690.1772773

[24] Ruslan Salakhutdinov and Andriy Mnih. 2007. Probabilistic matrix factorization. In Proceedings of the 20th International Conference on Neural Information Processing Systems (NIPS'07). 1257-1264. http://papers.nips.cc/paper/ 3208-probabilistic-matrix-factorization.

[25] Yang Song, Ali Mamdouh Elkahky, and Xiaodong He. 2016. Multi-rate deep learning for temporal recommendation. In Proceedings of the 39th International ACM SIGIR Conference on Research and Development in Information Retrieval (SIGIR'16). 909-912. DOI : https://doi.org/10.1145/2911451.2914726

[26] Hao Wang, Yanmei Fu, Qinyong Wang, Hongzhi Yin, Changying Du, and Hui Xiong. 2017. A location-sentimentaware recommender system for both home-town and out-of-town users. In Proceedings of the23rd ACM SIGKDD International Conference on Knowledge Discovery and Data Mining (KDD'17). 1135-1143. DOI : https://doi.org/10.1145/ 3097983.3098122

[27] Carl Yang, Lanxiao Bai, Chao Zhang, Quan Yuan, and Jiawei Han. 2017. Bridging collaborative filtering and semisupervised learning: A neural approach for POI recommendation. In Proceedings of the 23rd ACM SIGKDD International Conference on Knowledge Discovery and Data Mining (KDD'17). 1245-1254. DOI:https://doi.org/10.1145/ 3097983.3098094

[28] Cheng Yang, Maosong Sun, Wayne Xin Zhao, Zhiyuan Liu, and Edward Y. Chang. 2017. A neural network approach to jointly modeling social networks and mobile trajectories. ACM Transactions on Information Systems 35, 4 (2017), Article 36, 28 pages. DOI : https://doi.org/10.1145/3041658

[29] Dingqi Yang, Daqing Zhang, Zhiyong Yu, and Zhu Wang. 2013. A sentiment-enhanced personalized location recommendation system. In Proceedings of the 24th ACM Conference on Hypertext and Social Media (HT'13). 119-128. DOI : https://doi.org/10.1145/2481492.2481505

[30] Mao Ye, Peifeng Yin, and Wang-Chien Lee. 2010. Location recommendation for location-based social networks. In Proceedings of the 18th SIGSPATIAL International Conference on Advances in Geographic Information Systems (GIS'10) 458-461. DOI : https://doi.org/10.1145/1869790.1869861

[31] Hongzhi Yin, Bin Cui, Xiaofang Zhou, Weiqing Wang, Zi Huang, and Shazia W. Sadiq. 2016. Joint modeling of user check-in behaviors for real-time point-of-interest recommendation. ACM Transactions on Information Systems 35, 2 (2016), Article 11, 44 pages. DOI : https://doi.org/10.1145/2873055

[32] Quan Yuan, Gao Cong, Zongyang Ma, Aixin Sun, and Nadia Magnenat-Thalmann. 2013. Time-aware point-of-interest recommendation. In Proceedings of the 36th International ACM SIGIR Conference on Research and Development in Information Retrieval (SIGIR'13). 363-372. DOI : https://doi.org/10.1145/2484028.2484030

[33] Quan Yuan, Gao Cong, and Aixin Sun. 2014. Graph-based point-of-interest recommendation with geographical and temporal influences. In Proceedings of the 23rd ACM International Conference on Information and Knowledge Management (CIKM'14). 659-668. DOI : https://doi.org/10.1145/2661829.2661983 
[34] Wei Zhang and Jianyong Wang. 2015. Location and time aware social collaborative retrieval for new successive pointof-interest recommendation. In Proceedings of the 24th ACM International Conference on Information and Knowledge Management (CIKM'15). 1221-1230. DOI : https://doi.org/10.1145/2806416.2806564

[35] Yuyu Zhang, Hanjun Dai, Chang Xu, Jun Feng, Taifeng Wang, Jiang Bian, Bin Wang, and Tie-Yan Liu. 2014. Sequential click prediction for sponsored search with recurrent neural networks. In Proceedings of the 28th AAAI Conference on Artificial Intelligence (AAAI'14). 1369-1375. http://www.aaai.org/ocs/index.php/AAAI/AAAI14/paper/view/8529.

[36] Yongfeng Zhang, Guokun Lai, Min Zhang, Yi Zhang, Yiqun Liu, and Shaoping Ma. 2014. Explicit factor models for explainable recommendation based on phrase-level sentiment analysis. In Proceedings of the 37th International ACM SIGIR Conference on Research and Development in Information Retrieval (SIGIR'14). 83-92. DOI : https://doi.org/10.1145/ 2600428.2609579

[37] Zhiqian Zhang, Chenliang Li, Zhiyong Wu, Aixin Sun, Dengpan Ye, and Xiangyang Luo. 2017. NEXT: A neural network framework for next POI recommendation. arXiv:1704.04576.

[38] Kaiqi Zhao, Gao Cong, Quan Yuan, and Kenny Q. Zhu. 2015. SAR: A sentiment-aspect-region model for user preference analysis in geo-tagged reviews. In Proceedings of the 2015 IEEE 31st International Conference on Data Engineering. 675-686. DOI : https://doi.org/10.1109/ICDE.2015.7113324

[39] Zhe Zhao, Tao Liu, Shen Li, Bofang Li, and Xiaoyong Du. 2017. Guiding the training of distributed text representation with supervised weighting scheme for sentiment analysis. Data Science and Engineering 2, 2 (2017), 178-186.

[40] Bolong Zheng, Han Su, Wen Hua, Kai Zheng, Xiaofang Zhou, and Guohui Li. 2017. Efficient clue-based route search on road networks. IEEE Transactions on Knowledge and Data Engineering 29, 9 (2017), 1846-1859.

[41] Bolong Zheng, Han Su, Kai Zheng, and Xiaofang Zhou. 2016. Landmark-based route recommendation with crowd intelligence. Data Science and Engineering 1, 2 (2016), 86-100.

[42] Kaiping Zheng, Wei Wang, Jinyang Gao, Kee Yuan Ngiam, Beng Chin Ooi, and James Wei Luen Yip. 2017. Capturing feature-level irregularity in disease progression modeling. In Proceedings of the 26th International Conference on Information and Knowledge Management (CIKM'17). 1579-1588. DOI : https://doi.org/10.1145/3132847.3132944

[43] Kai Zheng, Yu Zheng, Nicholas Jing Yuan, Shuo Shang, and Xiaofang Zhou. 2014. Online discovery of gathering patterns over trajectories. IEEE Transactions on Knowledge and Data Engineering 26, 8 (2014), 1974-1988. DOI : https://doi.org/10.1109/TKDE.2013.160

[44] Nan Zheng and Qiudan Li. 2011. A recommender system based on tag and time information for social tagging systems. Expert Systems with Applications 38, 4 (2011), 4575-4587. DOI : https://doi.org/10.1016/j.eswa.2010.09.131

[45] Yu Zheng. 2015. Trajectory data mining: An overview. ACM Transactions on Intelligent Systems and Technology 6, 3 (2015), Article 29, 41 pages. DOI : https://doi.org/10.1145/2743025

Received July 2018; revised November 2018; accepted February 2019 